\title{
Storm Track Shifts under Climate Change: What Can Be Learned from Large-Scale Dry Dynamics
}

\author{
CHEIKH MBengue AND TAPIO SCHNEIDER \\ California Institute of Technology, Pasadena, California, and Swiss Federal Institute of Technology Zurich, Zurich, Switzerland
}

(Manuscript received 10 July 2013, in final form 2 October 2013)

\begin{abstract}
Earth's storm tracks are instrumental for transporting heat, momentum, and moisture and thus strongly influence the surface climate. Climate models, supported by a growing body of observational data, have demonstrated that storm tracks shift poleward as the climate warms. But the dynamical mechanisms responsible for this shift remain unclear. To isolate what portion of the storm track shift may be accounted for by large-scale dry dynamics alone, disregarding the latent heat released in phase changes of water, this study investigates the storm track shift under various kinds of climate change in an idealized dry general circulation model (GCM) with an adjustable but constant convective stability. It is found that increasing the mean surface temperature or the convective stability leads to poleward shifts of storm tracks, even if the convective stability is increased only in a narrow band around the equator. Under warming and convective stability changes roughly corresponding to a doubling of $\mathrm{CO}_{2}$ concentrations from a present-day Earthlike climate, storm tracks shift about $0.8^{\circ}$ poleward, somewhat less than but in qualitative agreement with studies using moist GCMs. About $63 \%\left(0.5^{\circ}\right)$ of the poleward shift is shown to be caused by tropical convective stability variations. This demonstrates that tropical processes alone (the increased dry static stability of a warmer moist adiabat) can account for part of the poleward shift of storm tracks under global warming. This poleward shift generally occurs in tandem with a poleward expansion of the Hadley circulation; however, the Hadley circulation expansion does not always parallel the storm track shift.
\end{abstract}

\section{Introduction}

The cyclones and anticyclones that carry out the bulk of the heat, moisture, and momentum transport in Earth's extratropical atmosphere are concentrated in storm tracks: regions of enhanced eddy kinetic energy (EKE) in the midlatitudes (Blackmon 1976; Blackmon et al. 1977). Storm tracks are found in the Northern Hemisphere primarily over the Atlantic and Pacific Oceans; in the Southern Hemisphere, they are more zonally uniform. In both hemispheres, the storm tracks, identified as regions of enhanced eddy kinetic energy, are currently centered around $50^{\circ}$ latitude. There is clear modeling evidence that storm tracks shift poleward as the climate warms globally, on average by about $2^{\circ}$ latitude under a doubling of carbon dioxide concentrations (Yin 2005; Bengtsson et al. 2006; Swart and Fyfe 2012; Barnes and

Corresponding author address: Cheikh Mbengue, Swiss Federal Institute of Technology Zurich-Geological Institute, Sonneggstrasse 5, 8092 Zurich, Switzerland.

E-mail: cmbengue@caltech.edu
Polvani 2013). However, it is unclear what dynamical processes are responsible for this poleward shift.

Numerous mechanisms driving a poleward shift of storm tracks have been proposed. One group of studies posits that low-level baroclinicity controls where storm tracks are located and that changes in it drive storm track shifts. For example, Yin (2005) suggests that a change in the meridional temperature gradients is responsible for a poleward shift of the maximum of a baroclinicity measure, implying a concomitant shift of storm tracks. By contrast, Lu et al. (2010) hold that an increase in the subtropical and midlatitude static stability is responsible for a poleward shift of the maximum of a similar baroclinicity measure and hence for a poleward shift of storm tracks. Furthermore, Brayshaw et al. (2008) posit that the strength and location of anomalous SST gradients relative to the location of the subtropical jet drive the poleward shift of storm tracks, while Butler et al. $(2010,2011)$ emphasize the role of tropical heating in modifying the low-level baroclinicity and storm track position. Another group of studies posits that changes in the upper troposphere and lower stratosphere modify 
wave propagation characteristics and thus lead to storm track shifts (e.g., Kushner and Polvani 2004; Chen and Held 2007; Lorenz and DeWeaver 2007; Chen et al. 2008; Butler et al. 2010). Yet others suggest that increasing eddy length scales under global warming may play a role in storm track shifts (Kidston et al. 2010; Rivière 2011).

These mechanisms are not mutually exclusive, and several of them may act simultaneously. Our goal in this paper is to disentangle distinct dynamical processes that may act jointly in global warming scenarios. For example, increased mean surface temperatures generally lead to a higher tropopause (Held 1982; Schneider 2007), to increased lower-level static stability through increased latent heat release in phase changes of water, both in the tropics and extratropics (e.g., Xu and Emanuel 1989; Emanuel 2007; Schneider and O'Gorman 2008), and typically to reduced pole-equator near-surface temperature contrasts through increased poleward latent heat transport (Pierrehumbert 2002; Caballero and Langen 2005; O'Gorman and Schneider 2008; Caballero and Hanley 2012). The presence of moisture in the atmosphere thus links surface temperature changes to static stability changes and to temperature gradient changes, which have distinct dynamical effects. To separate such effects, and to demonstrate that at least part of the poleward shift of storm tracks can be understood irrespective of how large-scale dynamics affect latent heat release, we study the storm track response to various idealized climate changes in a dry GCM, similar to those used by Kushner and Polvani (2004), Chen and Held (2007), and Butler et al. (2010). In the GCM, we vary the mean surface temperatures separately from an adjustable but constant convective stability, which controls the tropical static stability but has a less direct effect on the extratropical static stability (Schneider and Walker 2006; Schneider and O'Gorman 2008). This allows us to more clearly separate tropical from extratropical mechanisms driving storm track shifts.

\section{Idealized dry GCM and simulations}

We conduct a series of simulations over a wide range of climates using the idealized dry GCM described in detail in Schneider (2004) and Schneider and Walker (2006). Here, we give a brief overview of some aspects of the GCM pertinent to this study. The GCM uses the dry dynamical core of the Geophysical Fluid Dynamics Laboratory (GFDL)'s Flexible Modeling System and solves the primitive equations in a spherical shell with T85 horizontal resolution and 30 vertical sigma levels.

A Newtonian relaxation scheme represents radiative transfer and surface fluxes in an idealized way.
Temperatures are relaxed to a radiative equilibrium profile on a time scale of 7 days near the surface in low latitudes and 50 days in the rest of the atmosphere. The radiative equilibrium temperature at the surface is given by

$$
T_{s}^{e}(\phi)=\bar{T}_{s}^{e}+\Delta_{h}\left(\frac{1}{3}-\sin ^{2} \phi\right),
$$

where $\phi$ is latitude, $\Delta_{h}$ is the pole-equator thermal contrast, and $\bar{T}_{s}^{e}$ is the mean near-surface temperature in radiative equilibrium. The radiative equilibrium temperature away from the surface is given by

$$
T^{e}(\phi, p)=T_{t}^{e}\left[1+d_{0}(\phi)\left(\frac{p}{p_{0}}\right)^{\alpha}\right]^{1 / 4},
$$

where

$$
d_{0}(\phi)=\left[\frac{T_{s}^{e}(\phi)}{T_{t}^{e}}\right]^{4}-1
$$

is a latitude-dependent optical thickness that depends on the near-surface radiative equilibrium temperature $T_{s}^{e}(\phi)$. The optical depth is chosen so that the radiative equilibrium temperature $T_{t}^{e}$ at the top of the atmosphere is constant. In this study, it is set to $T_{t}^{e}=200 \mathrm{~K}$ in all simulations; that is, we assume a fixed longwave emission temperature, as the longwave emission temperature for a gray atmosphere is proportional to the temperature at the top of the atmosphere (Schneider 2007). The ratio $\alpha$ of the pressure scale height to the partial pressure scale height of the dominant infrared absorber controls the radiative equilibrium lapse rate [see Schneider (2004) for a discussion of the role of optical thickness and $\alpha$ in this GCM]. The pole-equator thermal contrast $\Delta_{h}$ is set to $120 \mathrm{~K}$, which is prescribed in the GCM and is chosen to ensure sufficient (Earth like) macroturbulence in midlatitudes.

We use standard Earth values for physical parameters. There are no continents in the GCM, and moisture effects, such as latent heat release and transport, are neglected. The radiative equilibrium profile of the dry GCM is unstable to thermal convection. The ensuing convection is represented by a quasi-equilibrium scheme, which relaxes temperatures in an atmospheric column toward a convective lapse rate given by $\gamma \Gamma_{d}$, whenever the column is less stable than one with this convective lapse. Here, $\Gamma_{d}=g / c_{p}$ is the dry adiabatic lapse rate, and $\gamma$ is a rescaling parameter, which is inversely proportional to the local degree of stability. Decreasing $\gamma$ increases the stability of the atmosphere, mimicking some of the effects of latent heat release in moist convection, 
except that the constant $\gamma$ is an adjustable model parameter that does not depend, for example, on temperature (Schneider 2004; Schneider and Walker 2006). The convection scheme essentially sets the lapse rate in the tropics; it does not control but can influence the lapse rate in the extratropics, particularly in weakly baroclinic climates or when the rescaling parameter $\gamma$ is small (Schneider and Walker 2006; Schneider and O'Gorman 2008).

In this study, we vary the rescaling parameter $\gamma$ to study the effect of the convective lapse rate on storm track position. To disentangle the effect of convective lapse rate changes that are global from those that are confined to the tropics, we consider two scenarios. 1) We use a constant rescaling parameter $\gamma$ globally and vary that. 2) We use an equatorial rescaling parameter $\gamma_{e}$ within $10^{\circ}$ of the equator and an off-equatorial parameter $\gamma_{x}$ outside this equatorial band, and we set $\gamma_{x}=1$ and vary $\gamma_{e}$. The second scenario resembles in some aspects the tropical heating simulations of Butler et al. (2010), but here we do not provide an additional enthalpy source to the tropical atmosphere, which would directly modify meridional temperature gradients.

Our goal is to investigate the mechanisms that may contribute to the poleward shift of storm tracks under global warming. Surface temperature increases have a number of consequences, among them an increase in the height of the tropopause if lapse rates remain fixed or decrease [see Held (1982) or, for a review, Schneider (2007)]. To study such effects separately, we perform two sets of simulations. In the first set, we vary the mean radiative equilibrium surface temperature $\bar{T}_{s}^{e}$ from 270 to $365 \mathrm{~K}$ in increments of $5 \mathrm{~K}$, implying an increasing optical thickness from (3) in radiative equilibrium, as the temperature at the top of the atmosphere $T_{t}^{e}$ is kept fixed. (While absolute temperatures do not affect dry dynamics, increasing the surface temperature in radiative equilibrium increases; for example, the tropopause height because the temperature at the top of the atmosphere $T_{t}^{e}$ is kept fixed.) We perform this set of simulations for fixed convective lapse rates that are either globally constant or that assume different values near the equator and away from it (see Table 1), with the lower convective rescaling parameter $\gamma=0.7$ roughly giving present-day moist-adiabatic convective lapse rates. In the second set of simulations, we keep the mean radiative equilibrium surface temperature fixed $\left(\bar{T}_{s}^{e}=340 \mathrm{~K}\right)$ and vary the convective lapse rate by varying the rescaling parameter in increments of 0.02 , either globally or varying it only near the equator while keeping $\gamma_{x}=1$ fixed (see Table 1). All simulations are spun up for two years, after which flow variables are sampled four times daily over a further two years and are then temporally and zonally averaged.
TABLE 1. Simulation parameters (surface temperature ranges are incremented by $5 \mathrm{~K}$ and convective lapse rate ranges are incremented by $0.2 \mathrm{~K} \mathrm{~km}^{-1}$ ).

\begin{tabular}{cccc}
\hline $\begin{array}{c}\text { Convection } \\
\text { scenario }\end{array}$ & $\begin{array}{c}\text { Surface } \\
\text { temperature } \\
(\mathrm{K})\end{array}$ & $\begin{array}{c}\text { Tropical } \\
\text { convective lapse } \\
\text { rate }\left(\mathrm{K} \mathrm{km}^{-1}\right)\end{array}$ & $\begin{array}{c}\text { Extratropical } \\
\text { convective lapse } \\
\text { rate }\left(\mathrm{K} \mathrm{km}^{-1}\right)\end{array}$ \\
\hline $\begin{array}{c}\gamma_{e}=0.7, \\
\gamma_{x}=0.7\end{array}$ & $270-365$ & 6.9 & 6.9 \\
$\begin{array}{c}\gamma_{e}=0.7, \\
\gamma_{x}=1\end{array}$ & $270-365$ & 6.9 & 9.8 \\
$\begin{array}{c}\gamma_{e}=1, \\
\gamma_{x}=0.7\end{array}$ & $270-365$ & 9.8 & 6.9 \\
$\begin{array}{c}\gamma_{e}=1, \\
\gamma_{x}=1\end{array}$ & $270-365$ & 9.8 & 9.8 \\
$\begin{array}{c}\gamma_{e} \text { variable, } \\
\gamma_{x}=1\end{array}$ & 340 & $6.0-9.8$ & 9.8 \\
$\begin{array}{c}\gamma_{x} \text { variable, } \\
\gamma_{x} \text { variable }\end{array}$ & 340 & $6.0-9.8$ & $6.0-9.8$ \\
\hline
\end{tabular}

\section{Simulation results}

Figures 1 and 2 show a sample of the simulated climates at the extremes of the scenarios investigated. It is apparent from the figures that the tropopause height and the subtropical jet speed increase with increasing radiative equilibrium temperature and increasing convective stability, as expected from radiative equilibrium considerations (Schneider 2007) and thermal wind shear extending over a deeper troposphere. The maximum of the eddy momentum flux convergence migrates poleward and upward. The Hadley cell strengthens with increasing radiative equilibrium temperature, but it weakens with increasing convective stability. The shallow recirculation in the streamfunction seen in Figs. 1c,d and 2c results from flow separation induced by an adverse pressure gradient caused by the change in the convective lapse rate across $10^{\circ}$ latitude (reversal of the near-surface meridional temperature gradient); however, our results are unaffected.

Table 2 (upper block of rows) and Fig. 3 show how the storm track location (as determined by the latitude of maximum barotropic EKE) responds to radiative equilibrium temperature changes. The storm tracks migrate poleward with increasing radiative equilibrium temperature in all convection scenarios; the magnitude of the poleward migration increases as the convective stability increases. These findings are qualitatively similar to those we obtain using other proxies for storm tracks, including extrema of the mean surface westerlies and of eddy heat fluxes (cf. Fig. 1). It is interesting that all storm track proxies studied migrate poleward in the statically neutral convection scenario: $\gamma_{e}=1, \gamma_{x}=1$. This shows that changes in moist processes are not a prerequisite for poleward migration of storm tracks. Dry dynamics can explain part of the storm track response to warming. It 

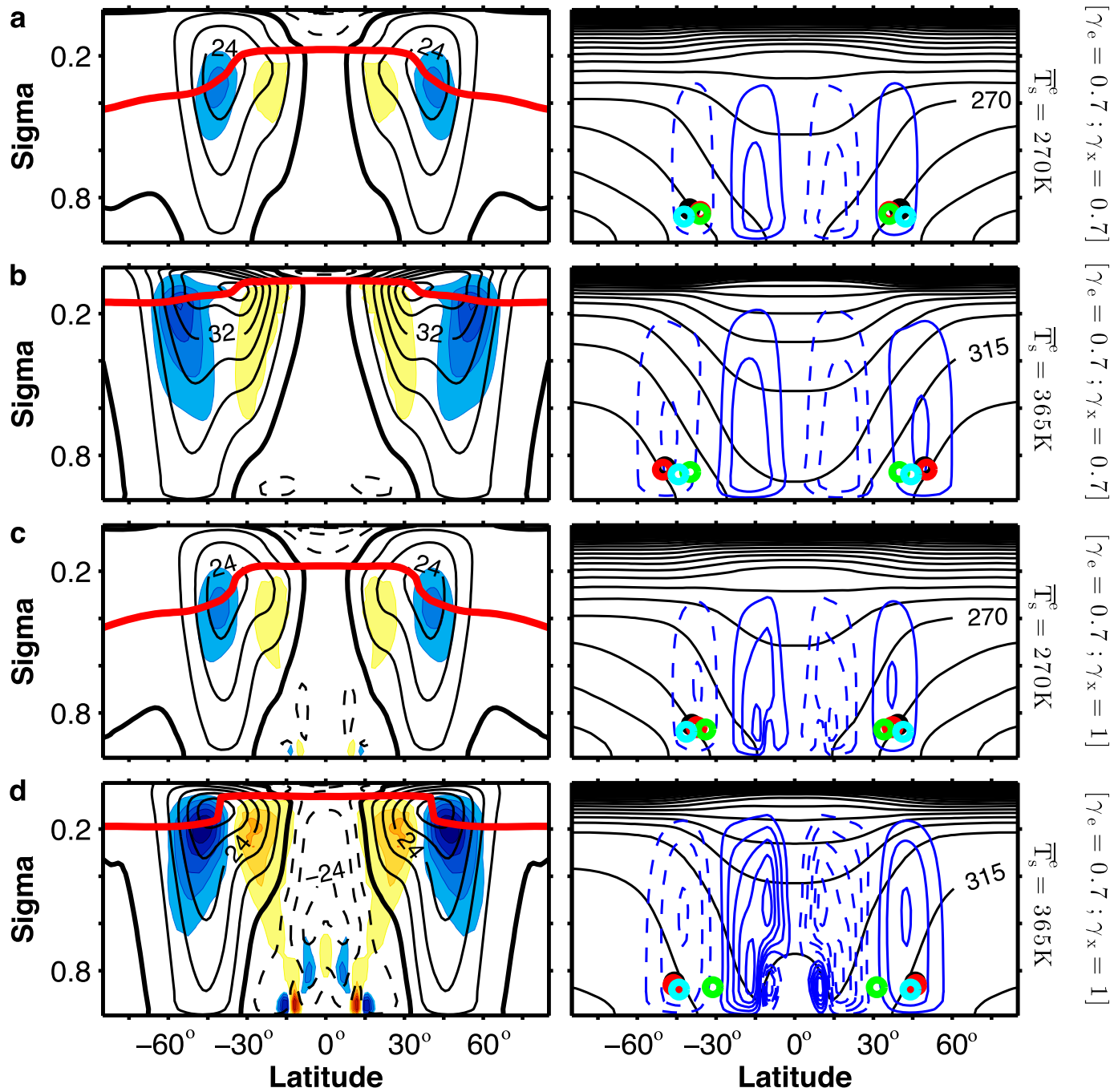

FIG. 1. Sample of simulated climates as the radiative equilibrium surface temperature is varied: (a) coldest climate with $\gamma=0.7$ globally, (b) warmest climate with $\gamma=0.7$ globally, (c) coldest climate with $\gamma_{e}=0.7$ and $\gamma_{x}=1$, and (d) warmest climate with $\gamma_{e}=0.7$ and $\gamma_{x}=1$. (left) Mean zonal wind (black contours with interval $8 \mathrm{~m} \mathrm{~s}^{-1}$ ), eddy momentum flux divergence (filled contours with interval $17 \times 10^{-6} \mathrm{~m} \mathrm{~s}^{-2}$; yellow is positive), and tropopause [thick red line, World Meteorological Organization (WMO) criterion]. (right) Potential temperature (black contours with interval $15 \mathrm{~K}$ ), mass flux streamfunction (blue contours with interval $30 \times 10^{9} \mathrm{~kg} \mathrm{~s}^{-1}$ ). Comparing (a) with (b) and (c) with (d) shows the response to mean radiative equilibrium temperature changes. The circles show the location of the storm tracks as indicated by extrema in barotropic eddy kinetic energy (red), meridional eddy potential temperature flux (green), vertical heat flux (cyan), and surface westerlies (black).

also shows that a fraction of the poleward migration of the storm tracks is independent of convective stability variations.

Changes in the height of the tropopause have been cited as a primary cause of the poleward migration of storm tracks (Williams 2006; Lorenz and DeWeaver 2007). We also find that increasing the radiative equilibrium temperature leads to a rise in the height of the tropopause and a poleward migration of storm tracks (see also Schneider 2004). It is likely that the rising tropopause facilitates the dynamics necessary for a poleward migration of storm tracks, but the causality is not obvious. Furthermore, counterexamples exist; for example, during El Niño events (narrow tropical heating), the tropopause height increases while the storm tracks move equatorward. In this situation, tropical temperature gradient changes dominate over stability changes, leading to an equatorward shift of the jets and a contraction of the Hadley circulation (Seager et al. 2003; Tandon et al. 2012).

Figure 4 shows the response of barotropic EKE to increasing tropical and global convective stability. The 
a
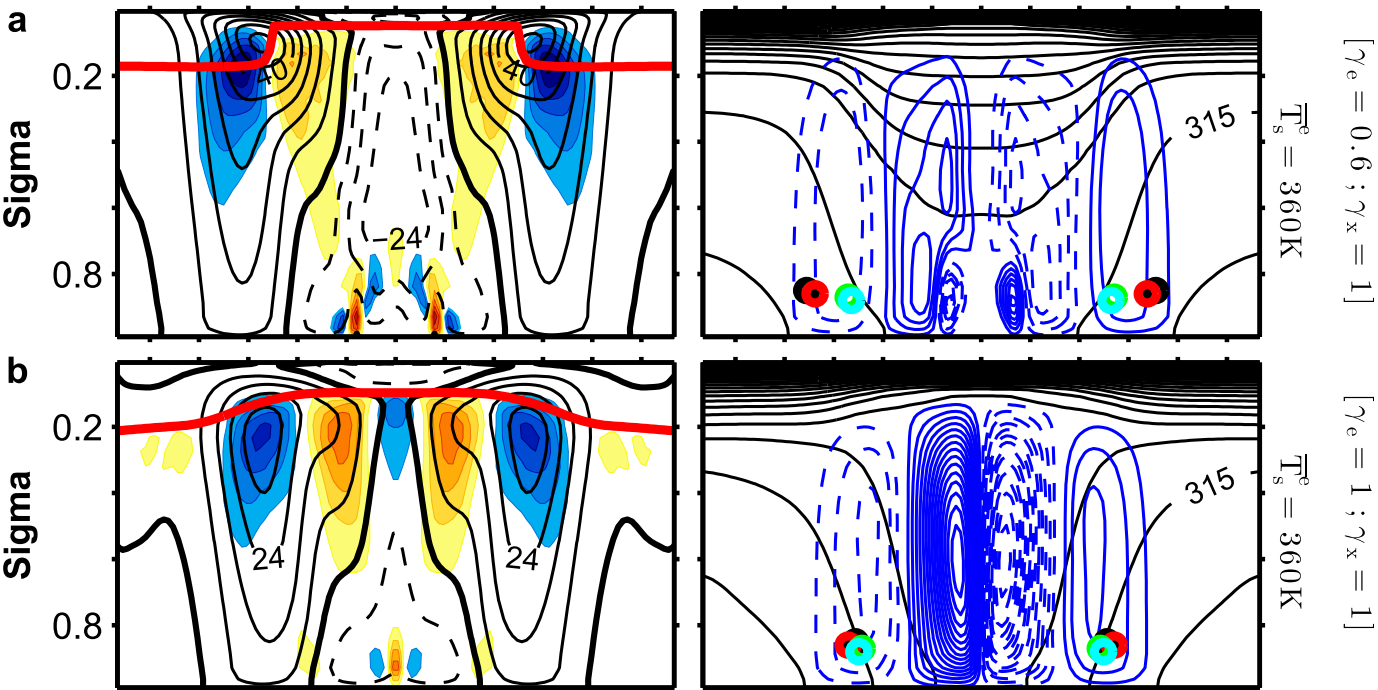

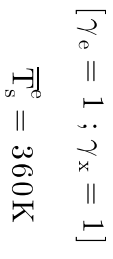
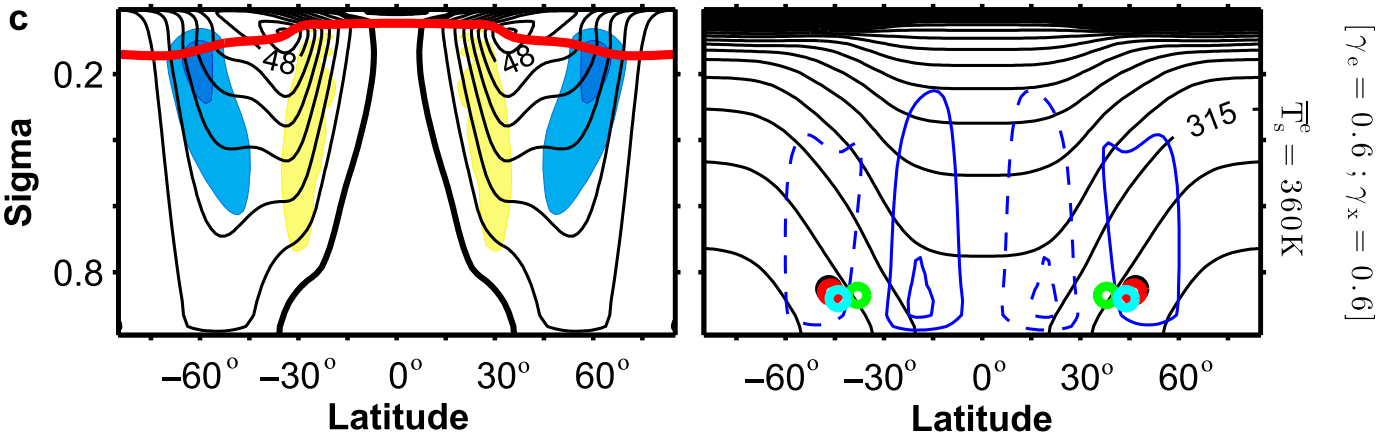

FIG. 2. Sample of simulated climates as the convective stability is varied. (a) Most stable and (b) least stable climates with variable $\gamma_{e}$ and $\gamma_{x}=1$. (c) Most stable climate with globally uniform convective stability, $\gamma=0.6$. Plotting conventions are as in Fig. 1 except the contour interval of eddy momentum flux divergence is $18 \times$ $10^{-6} \mathrm{~m} \mathrm{~s}^{-2}$. Compare (a) to (b) for the response to global convective stability changes and (b) to (c) for the response to tropical convective stability changes.

results show that the storm tracks generally migrate poleward with increasing global and tropical convective stability. On the interval $0.8 \lesssim \gamma_{e} \lesssim 0.9$, the storm track migration is essentially the same (a linear regression showing about $2^{\circ}$ poleward per $1 \mathrm{~K} \mathrm{~km}^{-1}$ ) whether or not the extratropical convective stability $\left(\gamma_{x}\right)$ is varied along with the tropical convective stability $\left(\gamma_{e}\right)$.
For $\gamma_{e} \lesssim 0.8$ (which include Earthlike climates), the poleward migration is enhanced by a further $1^{\circ}$ latitute per $1 \mathrm{~K} \mathrm{~km}^{-1}$ if $\gamma_{x}$ is varied simultaneously with $\gamma_{e}$, showing the increasing influence of convection on the extratropical static stability and the storm track response (Schneider and Walker 2006; Schneider and O’Gorman 2008).

TABLE 2. Storm track response to variations in mean radiative equilibrium temperature (top four rows) and convective stability (bottom two rows). Columns from left to right are the convection scenario, the latitude of the storm tracks in the coldest and warmest climate, the latitude of the storm tracks in the least and most stable climate, the total storm track latitudinal migration over the climate range, and the maximum storm track excursion (the difference between the maximum and minimum latitudes reached by the storm tracks).

\begin{tabular}{|c|c|c|c|c|c|c|}
\hline Convection scenario & Coldest & Warmest & Least stable & Most stable & Migration & Maximum excursion \\
\hline$\gamma_{e}=0.7, \gamma_{x}=0.7$ & $36^{\circ}$ & $50^{\circ}$ & - & - & $14^{\circ}$ & $14^{\circ}$ \\
\hline$\gamma_{e}=0.7, \gamma_{x}=1$ & $34^{\circ}$ & $47^{\circ}$ & - & - & $13^{\circ}$ & $14^{\circ}$ \\
\hline$\gamma_{e}=1, \gamma_{x}=0.7$ & $37^{\circ}$ & $46^{\circ}$ & - & - & $9^{\circ}$ & $9^{\circ}$ \\
\hline$\gamma_{e}=1, \gamma_{x}=1$ & $33^{\circ}$ & $42^{\circ}$ & - & - & $9^{\circ}$ & $9^{\circ}$ \\
\hline$\gamma_{e}$ variable, $\gamma_{x}=1$ & - & - & $41^{\circ}$ & $46^{\circ}$ & $6^{\circ}$ & $6^{\circ}$ \\
\hline$\gamma_{e}$ variable, $\gamma_{x}$ variable & - & - & $41^{\circ}$ & $50^{\circ}$ & $10^{\circ}$ & $11^{\circ}$ \\
\hline
\end{tabular}



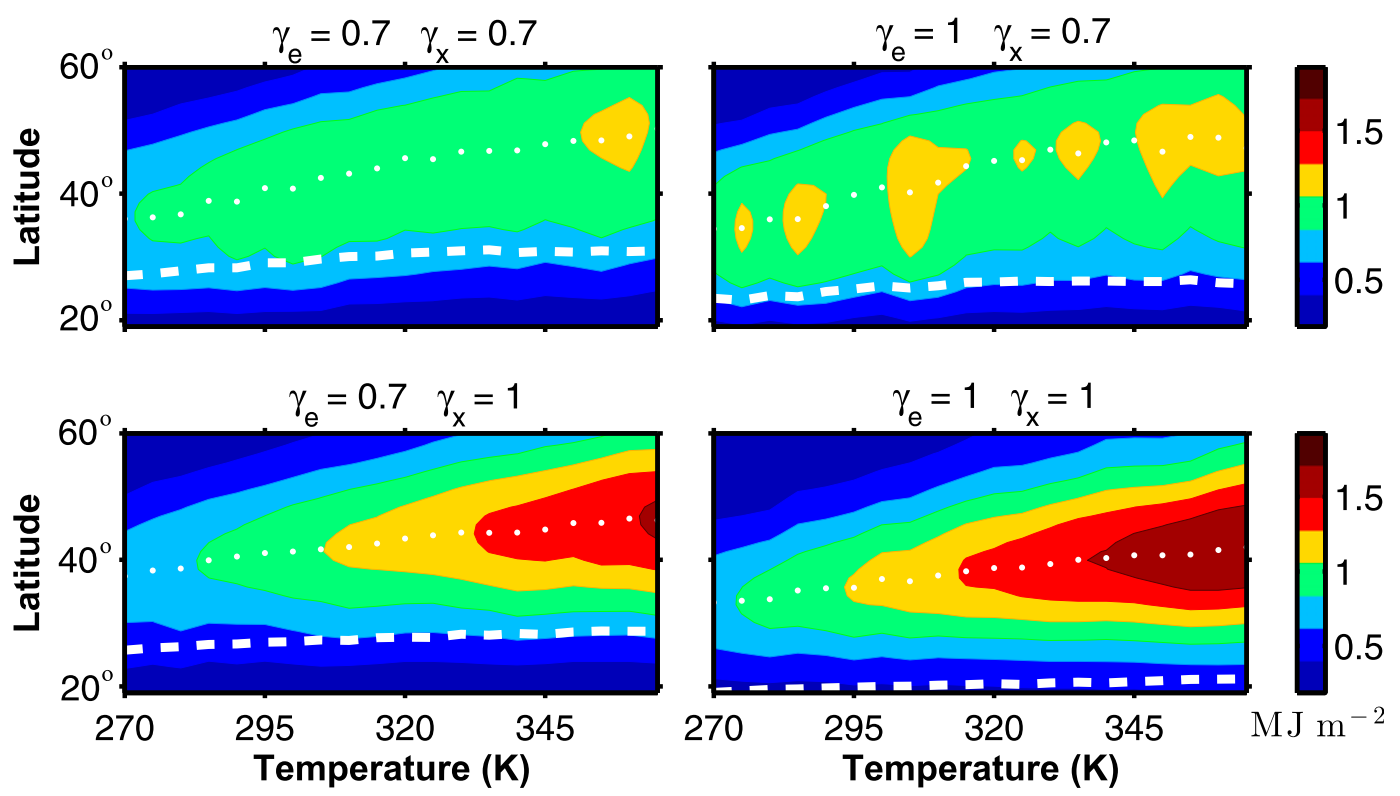

FIG. 3. Barotropic eddy kinetic energy as a function of latitude plotted across climates with increasing mean surface temperature in radiative equilibrium. The convection scenario is stated above each plot. Here, $\gamma_{e}$ is the convective stability rescaling parameter within $\pm 10^{\circ}$ of the equator; $\gamma_{x}$ is the value outside of this latitude band. The white dots show the EKE maxima, each marking the storm track location in the respective climate. The thick white dashed line shows the terminus of the Hadley cell, defined as the latitude at which the Eulerian mass streamfunction changes sign at the altitude where it achieves its extremum.

Previous studies have shown a dependence of storm track response on storm track latitude (Kidston and Gerber 2010; Garfinkel et al. 2012). This result is qualitatively reproduced in our simulations varying convective stability (Fig. 4) and varying radiative equilibrium temperatures (top right panel of Fig. 3): the storm track's poleward migration levels off at the highest latitudes. Additionally, storm tracks shift little as the convective stability is varied for $\gamma \gtrsim 0.9$; the reasons for this are unclear.

All simulations show that the Hadley circulation widens as the storm tracks migrate poleward (Figs. 3 and 4) - a correlation noted previously by Kang and Polvani (2010) and Ceppi and Hartmann (2012). But Fig. 4 shows more particularly that storm tracks migrate in tandem with the Hadley cell terminus when varying only the tropical convective stability; however, the migration is less parallel when the convective stability is varied globally. Nonetheless, this suggests that the Hadley cell is responsible for communicating the variations in tropical convective stability to the storm tracks in the midlatitudes. The role of the tropical convective stability in eliciting a response in the midlatitude storm tracks has not been previously identified. This result complements the results found by Butler et al. (2010). It also raises questions about the mechanisms facilitating the requisite tropical-extratropical interactions.

Using our simulations, we estimate the individual contributions of changes in radiative equilibrium temperature and tropospheric stability to the storm track
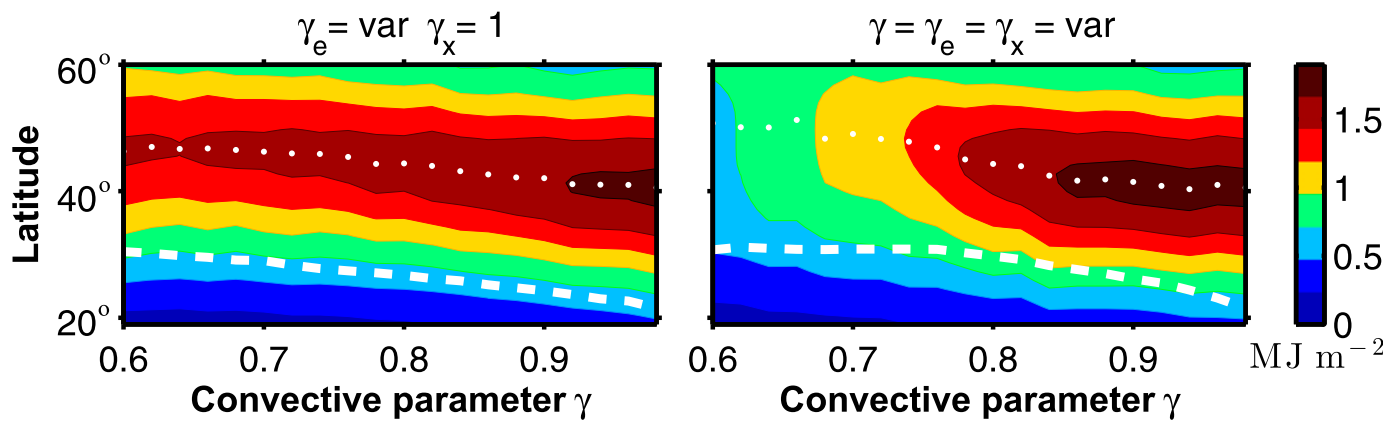

FIG. 4. As in Fig. 3, but showing storm track response to convective stability variations. 
response in a scenario similar to doubling $\mathrm{CO}_{2}$ concentration on Earth. To do so, we assume a $287-\mathrm{K}$ radiative equilibrium temperature with $\gamma_{e}=0.7$ and $\gamma_{x}=1$ as the initial Earthlike conditions. Then, we assume a canonical 3-K global-mean temperature increase when $\mathrm{CO}_{2}$ is doubled. The mean temperature sensitivity is obtained from the bottom left panel of Fig. 3 as the difference between the storm track latitudes $\phi(290 \mathrm{~K})$ and $\phi(287 \mathrm{~K})$. We use cubic spline interpolation to infer a $0.3^{\circ}$ poleward migration for the $3-\mathrm{K}$ mean temperature change.

Sensitivity to changes in tropospheric stability requires the change in $\gamma$ with a doubling of $\mathrm{CO}_{2}$. We use the Clausius-Clapeyron relation to find the saturation vapor pressure and mixing ratio at a reference pressure $(1000 \mathrm{mb})$ and calculate the moist adiabatic lapse rate change going from 287 to $290 \mathrm{~K}$. This gives a decrease of $0.3 \mathrm{~K} \mathrm{~km}^{-1}$ in lapse rate, or a 0.03 decrease in $\gamma$. Finally, we obtain the shift from the left panel of Fig. 4 as the difference between the storm track latitudes $\phi\left(\gamma_{e}=\right.$ $\left.0.67, \gamma_{x}=1\right)$ and $\phi\left(\gamma_{e}=0.7, \gamma_{x}=1\right)$. This yields a $0.5^{\circ}$ poleward migration because of tropical convective stability changes. To a first order, we assume that the two responses are additive; thus, our dry framework predicts a $0.8^{\circ}$ poleward migration of the storm tracks under $\mathrm{CO}_{2}$ doubling. This is less than what is found in studies with more comprehensive GCMs, but it is of the same order of magnitude (Yin 2005; Tsushima et al. 2006; Schneider et al. 2010; Barnes and Polvani 2013). The discrepancy is likely a result of the linearity assumption made here, the neglect of either the extratropical static stability changes associated with global warming or the many feedbacks associated with global warming in moist atmospheres. Nonetheless, if we consider the jet shifts reported in Barnes and Polvani (2013) using simulations from phase 5 of the Coupled Model Intercomparison Project (CMIP5), our dry model accounts for $40 \%$ of the shift in the storm tracks seen in the Southern Hemisphere and for $80 \%$ of that in the Northern Hemisphere. The shift associated with tropical stability changes is found to be $2 / 3$ greater than the shift associated with mean temperature changes. This suggests that tropical stability changes are as important, if not more important.

\section{Conclusions}

We have presented a study that decouples mean temperature effects from convective stability effects and tropical effects from extratropical ones. We used an idealized framework to investigate the storm tracks' response to changes in the radiative equilibrium temperature and in the global and tropical convective stability, each varied independently. The storm tracks are found to generally migrate poleward with increasing radiative equilibrium temperature and with increasing convective stability, even in the absence of moisture effects and with fixed pole-equator thermal contrast in radiative equilibrium. Moreover, the storm tracks migrate poleward as the climate warms in the statically neutral scenario, meaning that dry dynamics may explain part of the storm track migration seen in moist models. Indeed, when our results are translated to what they imply for a moist framework, we found that the change in mean temperature alone accounts for roughly one-third of the storm tracks' poleward migration, while convective stability changes (i.e., changes in the moist adiabatic lapse rate) account for the rest. Tropical convective stability has a surprisingly large effect on the storm tracks, suggesting that tropical-extratropical interactions are important. It begs the question of how varying the convective stability in a $10^{\circ}$ zonal band around the equator leads to a poleward migration of the midlatitude storm tracks. One possibility is through the Hadley circulation, which was shown to expand in tandem with the storm tracks in many (but not all) of the simulations.

Acknowledgments. We are grateful for the financial support provided by the National Science Foundation (Grant AGS-1019211) and for helpful discussions with Xavier Levine.

\section{REFERENCES}

Barnes, E. A., and L. Polvani, 2013: Response of the midlatitude jets, and of their variability, to increased greenhouse gases in the CMIP5 models. J. Climate, 26, 7117-7135.

Bengtsson, L., K. I. Hodges, and E. Roeckner, 2006: Storm tracks and climate change. J. Climate, 19, 3518-3543.

Blackmon, M. L., 1976: A climatological spectral study of the $500-\mathrm{mb}$ geopotential height of the Northern Hemisphere. J. Atmos. Sci., 33, 1607-1623.

_ J. M. Wallace, N.-C. Lau, and S. L. Mullen, 1977: An observational study of the Northern Hemisphere wintertime circulation. J. Atmos. Sci., 34, 1040-1053.

Brayshaw, D. J., B. Hoskins, and M. Blackburn, 2008: The stormtrack response to idealized SST perturbations in an aquaplanet GCM. J. Atmos. Sci., 65, 2842-2860.

Butler, A. H., D. W. J. Thompson, and R. Heikes, 2010: The steadystate atmospheric circulation response to climate change-like thermal forcings in a simple general circulation model. J. Climate, 23, 3474-3496.

,$- \ldots$, and T. Birner, 2011: Isentropic slopes, downgradient eddy fluxes, and the extratropical atmospheric circulation response to tropical tropospheric heating. J. Atmos. Sci., 68, 2292-2305.

Caballero, R., and P. L. Langen, 2005: The dynamic range of poleward energy transport in an atmospheric general circulation model. Geophys. Res. Lett., 32, L02705, doi:10.1029/ 2004GL021581.

- and J. Hanley, 2012: Midlatitude eddies, storm-track diffusivity, and poleward moisture transport in warm climates. J. Atmos. Sci., 69, 3237-3250. 
Ceppi, P., and D. L. Hartmann, 2012: On the speed of the eddydriven jet and the width of the Hadley cell in the Southern Hemisphere. J. Climate, 26, 3450-3465.

Chen, G., and I. M. Held, 2007: Phase speed spectra and the recent poleward shift of Southern Hemisphere surface westerlies. Geophys. Res. Lett., 34, L21805, doi:10.1029/2007GL031200.

— J. Lu, and D. M. W. Frierson, 2008: Phase speed spectra and the latitude of surface westerlies: Interannual variability and global warming trend. J. Climate, 21, 5942-5959.

Emanuel, K., 2007: Quasi-equilibrium dynamics of the tropical atmosphere. The Global Circulation of the Atmosphere, T. Schneider and A. Sobel, Eds., Princeton University Press, 186-218.

Garfinkel, C. I., D. W. Waugh, and E. P. Gerber, 2012: The effect of tropospheric jet latitude on coupling between the stratospheric polar vortex and the troposphere. J. Climate, 26, 20772095.

Held, I. M., 1982: On the height of the tropopause and the static stability of the troposphere. J. Atmos. Sci., 39, 412-417.

Kang, S. M., and L. M. Polvani, 2010: The interannual relationship between the latitude of the eddy-driven jet and the edge of the Hadley cell. J. Climate, 24, 563-568.

Kidston, J., and E. P. Gerber, 2010: Intermodel variability of the poleward shift of the austral jet stream in the CMIP3 integrations linked to biases in 20th century climatology. Geophys. Res. Lett., 37, L09708, doi:10.1029/2010GL042873.

, S. M. Dean, J. A. Renwick, and G. K. Vallis, 2010: A robust increase in the eddy length scale in the simulation of future climates. Geophys. Res. Lett., 37, L03806, doi:10.1029/ 2009GL041615.

Kushner, P. J., and L. M. Polvani, 2004: Stratosphere-troposphere coupling in a relatively simple AGCM: The role of eddies. J. Climate, 17, 629-639.

Lorenz, D. J., and E. T. DeWeaver, 2007: Tropopause height and zonal wind response to global warming in the IPCC scenario integrations. J. Geophys. Res., 112, D10119, doi:10.1029/ 2006JD008087.

Lu, J., G. Chen, and D. M. W. Frierson, 2010: The position of the midlatitude storm track and eddy-driven westerlies in aquaplanet AGCMs. J. Atmos. Sci., 67, 3984-4000.

O'Gorman, P. A., and T. Schneider, 2008: The hydrological cycle over a wide range of climates simulated with an idealized GCM. J. Climate, 21, 3815-3832.
Pierrehumbert, R. T., 2002: The hydrologic cycle in deep-time climate problems. Nature, 419, 191-198.

Rivière, G., 2011: A dynamical interpretation of the poleward shift of the jet streams in global warming scenarios. J. Atmos. Sci., 68, 1253-1272.

Schneider, T., 2004: The tropopause and the thermal stratification in the extratropics of a dry atmosphere. J. Atmos. Sci., 61, 1317-1340.

_ - 2007: The thermal stratification of the extratropical troposphere. The Global Circulation of the Atmosphere, T. Schneider and A. Sobel, Eds., Princeton University Press, 47-77.

— , and C. C. Walker, 2006: Self-organization of atmospheric macroturbulence into critical states of weak nonlinear eddyeddy interactions. J. Atmos. Sci., 63, 1569-1586.

_ , and P. A. O'Gorman, 2008: Moist convection and the thermal stratification of the extratropical troposphere. J. Atmos. Sci., 65, 3571-3583.

,$- \ldots$, and X. J. Levine, 2010: Water vapor and the dynamics of climate changes. Rev. Geophys., 48, RG3001, doi:10.1029/ 2009RG000302.

Seager, R., N. Harnik, Y. Kushnir, W. Robinson, and J. Miller, 2003: Mechanisms of hemispherically symmetric climate variability. J. Climate, 16, 2960-2978.

Swart, N. C., and J. C. Fyfe, 2012: Observed and simulated changes in the Southern Hemisphere surface westerly wind-stress. Geophys. Res. Lett., 39, L16711, doi:10.1029/2012GL052810.

Tandon, N. F., E. P. Gerber, A. H. Sobel, and L. M. Polvani, 2012: Understanding Hadley cell expansion versus contraction: Insights from simplified models and implications for recent observations. J. Climate, 26, 4304-4321.

Tsushima, Y., and Coauthors, 2006: Importance of the mixed-phase cloud distribution in the control climate for assessing the response of clouds to carbon dioxide increase: A multi-model study. Climate Dyn., 27, 113-126.

Williams, G. P., 2006: Circulation sensitivity to tropopause height. J. Atmos. Sci., 63, 1954-1961.

Xu, K.-M., and K. A. Emanuel, 1989: Is the tropical atmosphere conditionally unstable? Mon. Wea. Rev., 117, 1471-1479.

Yin, J. H., 2005: A consistent poleward shift of the storm tracks in simulations of 21st century climate. Geophys. Res. Lett., 32, L18701, doi:10.1029/2005GL023684. 